Michael Katzmayr* und Thomas Seyffertitz

\title{
Produktion sowie Nutzung von Forschungsdaten und das Datenmanagement Forschender: eine empirische Analyse
}

https://doi.org/10.1515/bfp-2021-0011

Zusammenfassung: Hochschulbibliotheken oder andere forschungsunterstützende Serviceabteilungen, die sich mit Forschungsdatenmanagement befassen, verfügen häufig nicht über umfassende Informationen darüber, welche Arten von Forschungsdaten an der jeweiligen Hochschule gesammelt, verwendet, produziert und verbreitet werden. Ein umfassender Überblick über die Forschungsdatenlandschaft ist Voraussetzung für die strategische Planung und Entwicklung geeigneter, lokal angepasster Serviceleistungen. Diese Fallstudie beschreibt die Erhebung der Produktion und Verwendung von Forschungsdaten an der Wirtschaftsuniversität Wien (WU) und darauf aufbauend die Etablierung und Koordination des Forschungsdatenmanagements durch die Universitätsbibliothek. Die Studie führte einerseits zu interessanten und für die Serviceentwicklung relevanten Ergebnissen. Andererseits wurde die hierbei verwendete Herangehensweise in ein Vorgehensmodell überführt, das auch an anderen wissenschaftlichen Einrichtungen anwendbar erscheint.

Schlüsselwörter: Forschungsdatenmanagement; Universität; Erhebung; Universitätsbibliothek

\section{Production and Use of Research Data and Researchers' Data Management: An Empirical Analysis}

Abstract: University libraries or other service departments involved in research data management often do not have comprehensive information about what types of research data are collected, used, produced, and disseminated within the institution concerned. A comprehensive overview of the research data landscape is a prerequisite for the strategic planning and development of suitable, locally adapted services. This case study describes the survey of the production and use of research data at the Vienna University of Economics and Business (WU) and, based on this, the establishment and coordination of research data management by the university library. On the one hand, the

*Kontaktperson: Michael Katzmayr, michael.katzmayr@wu.ac.at Thomas Seyffertitz, thomas.seyffertitz@wu.ac.at study led to interesting results relevant to service development. On the other hand, the approach used here was converted into a procedural model that also appears to be applicable to other academic institutions

Keywords: Research data management; university; empirical analysis; university library

Inhalt

1 Einleitung und Kontext der Fallstudie . . . . . . . 317

2 Forschungsdesign.................. 319

2.1 Fallstudienansatz . . . . . . . . . . . . . . . 319

2.2 Quantitative Analyse: Dokumentenanalyse der

Forschungsergebnisse . . . . . . . . . . 320

2.3 Qualitative Analyse: Semi-strukturierte Inter-

views ..................... 321

3 Ergebnisse ................... 322

3.1 Dokumentenanalyse . . . . . . . . . . . 322

3.2 Semi-strukturierte Interviews . . . . . . . . . . . 325

4 Diskussion und Umsetzung. . . . . . . . . . . . . 327

4.1 Zusammenfassung und Generalisierbarkeit der

Ergebnisse . . . . . . . . . . . . . . . . 327

4.2 Umsetzung . . . . . . . . . . . . . . . 328

4.3 Ableitung eines Vorgehensmodells im FDM. . . . . 329

5 Schlussfolgerungen und Ausblick . . . . . . . . 330

\section{Einleitung und Kontext der Fallstudie}

Der sich vollziehende digitale Wandel in Wissenschaft und Forschung ist $\mathrm{zu}$ einem wesentlichen Teil durch einen enormen Zuwachs an Menge und Bedeutung von Forschungsdaten gekennzeichnet. Das Wachstum von für die Forschung verfügbaren Daten in Bezug auf Größe und Vielfalt sowie die Fähigkeit, Daten aus mehreren Quellen zu verknüpfen, haben zu einem stetigen Anstieg datenintensiver Forschung geführt. Zusätzlich erzeugen und hinterlassen Forschungsprojekte selbst wiederum enorme Datenmengen, die einen Teil der Ergebnisse dar- 
stellen und zur Replikation bzw. Nachnutzung bereitstehen. ${ }^{1}$

Forschungsdaten können sehr breit definiert werden als all jene Daten, „die im Zuge wissenschaftlicher Vorhaben z. B. durch Digitalisierung, Quellenforschungen, Experimente, Messungen, Erhebungen oder Befragungen entstehen." ${ }^{2}$ Bereits publizierte Fachliteratur oder legislative Texte (z.B. Gesetzesmaterialien) zählen demnach üblicherweise nicht zu den Forschungsdaten im engeren Sinn. Eine Ausnahme besteht allerdings dann, wenn die publizierte Literatur direkt als Quelle für eine empirische Erhebung dient - etwa ein Sample an Aufsätzen im Rahmen systematischer Literaturreviews oder korpuslinguistische Analysen von Textsammlungen etc. Forschungsdatenmanagement (FDM) kann verstanden werden als

„Summe aller Strategien, Maßnahmen, Konventionen, Arbeitsabläufe etc., die den Umgang mit Forschungsdaten im Projektverlauf und darüber hinaus regeln, [es] beinhaltet auch organisatorische, technische und administrative Aspekte, die nicht explizit Gegenstand eines datenzentrierten Lebenszyklusmodells sind.“”

Als wesentliche Impulsgeber für das Aufkommen von FDM können gesehen werden:

1) Vor dem Hintergrund der Guten Wissenschaftlichen Praxis und der damit verbundenen Reproduzierbarkeit der Forschungsergebnisse werden Forschende zunehmend von Fördergebern und Verlagen verpflichtet, ihre dem Forschungsergebnis zugrundeliegenden Forschungsdaten (offen) zur Verfügung zu stellen. Verstärkt wird dieser Trend durch politische Initiativen auf nationaler oder europäischer Ebene, die regelmäßig von wissenschaftlichen Vereinigungen und bedeutenden Forschungsförderern mitgetragen werden. ${ }^{4}$

2) Datenschutzrechtliche Aspekte stellen die Forschungseinrichtungen mit dem Inkrafttreten der DatenschutzGrundverordnung (DSGVO) im Jahr 2018 vor eine große Herausforderung. Damit verbunden ist die Notwendigkeit der Sicherung von Forschungsdaten vor unbefugtem Zugriff und Datenverlust. ${ }^{5}$ Neben dem Datenschutzrecht sind insbesondere das Urheberrecht sowie auch das Patenrecht, das Arbeitnehmerrecht und Fragen zu Nutzungsrechten und Betriebsgeheimnissen in der Auftragsforschung zu nennen. Diese rechtlichen

1 Vgl. dazu Katerbow et al. (2020) 5.

2 Arbeitsgruppe Forschungsdaten (2018) 4.

3 Jensen (2019) 24.

4 Vgl. dazu ausführlicher etwa Van den Eynden und Corti (2020) $14 \mathrm{ff}$., Cox und Verbaan (2018) $41 \mathrm{ff}$.

$5 \mathrm{Vgl}$. Summers et al. (2020).
Fragen sind im Rahmen eines FDM zu berücksichtigen und zu regeln. ${ }^{6}$

Obwohl FDM eine vergleichsweise junge Geschichte hat, sind wissenschaftliche Bibliotheken in diesem Bereich naheliegende Dienstleister im Hochschulgefüge. Bestehende Kontakte zu den Forschenden sowie bereits vorhandene Serviceangebote wie Beratung und Unterstützung zu Open Access, der Betrieb von Repositorien sowie Kenntnisse in der inhaltlichen Beschreibung und im Umgang mit Metadaten bieten ideale Voraussetzungen, um sich in diesem Bereich zu positionieren. ${ }^{7}$ Hier spielen natürlich auch Erwägungen zur Zukunftssicherung der wissenschaftlichen Bibliotheken angesichts des ständigen Wandels in der Wissenschaftskommunikation eine Rolle. Wollen Bibliotheken mittelfristig nicht den Anschluss verlieren und als Lizenzierungsbüros oder Verwahrer alter Bücher übrig bleiben, so sind sie nicht zuletzt auch aus Eigeninteresse gefordert, sich hier als Dienstleister ins Spiel zu bringen. Dies ist auch eng verbunden mit der Entstehung neuer Berufsbilder in akademischen Bibliotheken, wie z.B. das des „Data Librarian“. 8

Vor diesem Hintergrund hat die Universitätsbibliothek der WU FDM als zukunftsträchtiges Themenfeld aufgegriffen. In der Folge wurde die Universitätsbibliothek vom Vizerektorat für Forschung beauftragt, ein Profil der Erzeugung und Nutzung von Forschungsdaten an der WU zu erstellen. Darüber hinaus sollte ein Einblick erlangt werden, wie die Forschenden verschiedener Fachbereiche tatsächlich mit ihren Forschungsdaten umgehen. Ebenso sollen Erfahrungen, Bedürfnisse und Trends in Bezug auf Forschungsdaten erhoben werden. Die dadurch gewonnenen Erkenntnisse sollten dazu dienen, im Rahmen der strategischen Serviceentwicklung möglichst passgenaue Angebote im Bereich FDM an der WU zu entwickeln.

Die WU ist eine öffentliche Universität und Österreichs einzige Wirtschaftsuniversität. Zu Beginn der empirischen Studie im Juni 2017 gab es kein institutionelles FDM. Mit Stichtag 31.12.2017 umfasste das wissenschaftliche Personal der WU 766,2 Vollzeitäquivalente (mit Stichtag 31.12. 2019: 780), die 11 akademischen Departments zugeordnet sind. Darüber hinaus gibt es derzeit 15 Forschungsinstitute und 7 Kompetenzzentren, die keiner bestimmten Abteilung zugeordnet sind. Die Forschungsschwerpunkte er-

\footnotetext{
6 Vgl. Johannes (2017) 901.

7 Vgl. Bertelmann und Pfeiffenberger (2015).

8 Vgl. Neuroth (2013).
} 
Tab. 1: Akademische Einheiten der WU, Stand März 2021

\begin{tabular}{lll}
\hline Departments & Forschungsinstitute & Kompetenzzentren \\
\hline Finance, Accounting and Statistics & Altersökonomie & Emerging Markets and CEE \\
Informationsverarbeitung und Prozessmanagement & Europafragen & Empirische Forschungsmethoden \\
Management & Familienunternehmen & Experimentalforschung \\
Marketing & Freie Berufe & Gründungszentrum \\
Strategy and Innovation & Internationale Besteuerung & Sustainability Transformation and Re- \\
Welthandel & Kooperationen und Genossenschaften & sponsibility (STaR) \\
Öffentliches Recht und Steuerrecht & Kryptoökonomie & Nonprofit-Organisationen und Social En- \\
Privatrecht & Mittel- und Osteuropäisches Wirtschaftsrecht & trepreneurship \\
Fremdsprachliche Wirtschaftskommunikation & Raum- und Immobilienwirtschaft & Zentrum für Wirtschaftssprachen \\
Sozioökonomie & Rechenintensive Methoden & \\
Volkswirtschaft & Regulierungsökonomie & \\
& Strategische Kapitalmarktforschung & \\
& Supply Chain Management & \\
& Urban Management and Governance & \\
\hline
\end{tabular}

geben sich im Wesentlichen aus der Aufbauorganisation bzw. dem Organisationsplan der WU (siehe Tab. 1). ${ }^{9}$

Der Beitrag ist wie folgt aufgebaut: Abschnitt 2 beschreibt das Forschungsdesign. Die Ergebnisse der quantitativen und qualitativen Analyse werden in Abschnitt 3 vorgestellt. Abschnitt 4 fasst diese zusammen, interpretiert die zentralen Erkenntnisse hinsichtlich möglicher Umsetzungsmaßnahmen und leitet ein konzeptionelles Vorgehensmodell ab, das als Ausgangspunkt für ähnliche Vorhaben dienen kann. Der abschließende Abschnitt bietet ein kurzes Resümee und liefert Hinweise auf weitere Modelle zur strategischen Planung von Forschungsdatenmanagement.

\section{Forschungsdesign}

\subsection{Fallstudienansatz}

Der Umgang mit Forschungsdaten ist nicht nur abhängig von der jeweiligen Fachdisziplin, sondern ganz wesentlich von nationalen und institutionellen Regelungen, die bestimmte Verhaltensanreize oder Verpflichtungen für Forschende setzen, etwa hinsichtlich der Wiederverwendbarkeit, der Reproduzierbarkeit bis hin zu der Verpflichtung der Veröffentlichung der verwendeten Forschungsdaten. Groß angelegte, multi-institutionelle oder national ausgerichtete Studien leisten einen wichtigen Beitrag, um das Forschungsfeld hierbei aufzubereiten, erlauben aber häufig keinen ausreichend detaillierten Einblick in die jeweili-

9 Vgl. WU Wirtschaftsuniversität Wien (2020). gen Institutionen. So wurden in einer groß angelegten österreichweiten Befragung ${ }^{10}$ zwar alle 21 öffentlichen Universitäten und drei außeruniversitäre Forschungseinrichtungen Österreichs erfasst, allerdings ließen sich daraus nur eingeschränkt und ansatzweise Erkenntnisse für Ausgestaltung des FDM an der WU ableiten.

Ergebnisse bereits vorhandener Studien anderer Institutionen können zwar ebenfalls interessante Ansatzpunkte zur Entwicklung maßgeschneiderter FDM-Services geben, können aber mangels Übertragbarkeit eben nur eingeschränkt auf andere Institutionen umgelegt werden. Der Nutzen bereits existierender Fallstudien für die eigene Institution ist noch am ehesten bei gleichen oder ähnlichen Fachdisziplinen gegeben. Grundsätzlich erfordert die Kontextbezogenheit der Thematik jedoch, dass im Rahmen der strategischen Serviceentwicklung jeweils die eigene Institution beforscht werden muss, um institutionelle Besonderheiten $\mathrm{zu}$ erfassen und diesen dann in weiterer Folge begegnen zu können. Der primäre wissenschaftliche Beitrag institutioneller Fallstudien im Bereich FDM liegt u.E. daher auch in der Offenlegung und Diskussion der methodischen Vorgehensweise, um sozusagen das Rad nicht jedes Mal erneu erfinden zu müssen. Hierin sehen wir auch einen ganz wesentlichen Beitrag der vorliegenden Studie.

Um somit einen möglichst detaillierten und facettenreichen Überblick über die Forschungsdatenlandschaft der WU zu erhalten und die spezifischen Forschungsdatenpraktiken $\mathrm{zu}$ untersuchen, ist ein institutioneller Fallstudienansatz das Vorgehen der Wahl. Dieses Unter-

10 Bauer et al. (2015). 
suchungsdesign ist immer dann angezeigt, wenn - wie im vorliegenden Fall - der Kontext für die Forschungsfrage von entscheidender Bedeutung ist und der Zweck der Forschung eine ganzheitliche, eingehende Untersuchung einer Situation aus Sicht der Stakeholder erfordert. ${ }^{11}$

Interessant in diesem Zusammenhang ist eine Untersuchung von Elizabeth Berman. ${ }^{12}$ Sie analysierte 23 publizierte Fallstudien zum FDM aus den Jahren 2007 bis 2016 und stellte fest, dass im überwiegenden Teil Interviews und schriftliche Befragungen als Erhebungsmethode zur Anwendung kamen, hingegen der Einsatz von Fokusgruppen und Dokumentenanalysen eine nachrangige Rolle spielten. Bis auf eine Ausnahme wurde in den Studien allerdings nur jeweils eine der erwähnten Methoden zur Erhebung verwendet - in lediglich einer Studie kamen zwei Methoden (Interviews und Fokusgruppen) zur Anwendung.

Das ist durchaus überraschend, immerhin stützt sich die Fallstudie als Forschungsstrategie üblicherweise auf mehrere Datenquellen und/oder Erhebungstechniken, um die Validität sicherzustellen. ${ }^{13}$ In der von Berman selbst durchgeführten institutionellen Fallstudie findet folgerichtig ebenso eine Kombination mehrerer Methoden (Interviews, Dokumentenanalyse, Umfrage) statt. ${ }^{14}$

Der Anforderung der methodischen Mehrfachabsicherung folgend wurde zunächst eine quantitative Dokumentenanalyse der Forschungsergebnisse der WU hinsichtlich der Verwendung und Generierung von Forschungsdaten durchgeführt. Anschließend kamen semi-strukturierte Interviews mit Forschenden zur Anwendung, um ein detaillierteres Verständnis der tatsächlichen Forschungsdatenpraktiken und der zugrundeliegenden Motivationen zu erhalten. In Gegensatz zur erwähnten Studie von Berman wurde an der WU die quantitative Erhebung zuerst durchgeführt, da die Ergebnisse der Dokumentenanalyse wichtige Ansatzpunkte für die Auswahl der Interviewpartner/ innen lieferten.

\subsection{Quantitative Analyse: Dokumentenanalyse der Forschungsergebnisse}

Die quantitative Analyse der wissenschaftlichen Zeitschriftenartikel widmete sich den Fragen, welche Arten und Typen von Forschungsdaten es an der WU gibt und mit

11 Vgl. Pickard und Chiles (2013).

12 Vgl. Berman (2017).

13 Vgl. Yin (2018).

14 Vgl. Berman (2017). welchen Daten vornehmlich gearbeitet wird. Die Auswertung sollte dabei helfen, einen Überblick über Einsatz, Entstehung und Verwendung von Forschungsdaten an der WU („Forschungsdatenprofil“) zu erreichen.

Abgefragt wurden dabei die Einträge aus dem universitären Forschungsinformationssystem in der Rubrik „Originalbeiträge in Fachzeitschrift“ für das gesamte Jahr 2016. Jeder Eintrag enthielt eine eindeutige Identifikationsnummer, die bibliografischen Angaben des Artikels, Name der Organisationseinheit (Department, Forschungsinstitut, Kompetenzzentrum) dem der Artikel zugeordnet wurde und sofern vorhanden eine DOI. Eine Überprüfung auf korrekte Zuordnung der Einträge zur Kategorie „Originalbeiträge in Fachzeitschrift“ wurde nicht vorgenommen. Zu Beginn enthielt der Datensatz insgesamt 774 Zeitschriftenartikel. Anhand der Identifikationsnummer konnten jene Artikel identifiziert werden, die im Datensatz mehrfach vorkommen, weil sie aufgrund der Autorenschaft sowohl einem Department als auch einem Forschungsinstitut zugeordnet wurden. Da in der vorliegenden Untersuchung als die kleinste Organisationsebene jene der Departments gewählt wurde, erfolgte die Zuordnung der 774 Einträge für das Ausgangssample nach den folgenden Regeln:

- Artikel, deren WU-Autor/inn/en aus verschiedenen Departments stammen, wurde jedem einmal zugeordnet.

- Artikel, die sowohl einem Department als auch einem Forschungsinstitut zugeordnet waren, wurden jenen Departments zugeordnet, aus welchem die jeweiligen Autor/inn/en stammen.

- Artikel, die ausschließlich einem Forschungsinstitut zugeordnet waren, wurden der Rubrik „sonstige“ zugeordnet, ebenso wie Artikel, die nicht zugeordnet waren.

Der bereinigte Datensatz enthielt 618 Einträge. ${ }^{15}$ Für die Auswertungen auf WU-Ebene wurden Artikeleinträge, die aufgrund von Ko-Autorenschaften mehreren Organisationseinheiten zugerechnet wurden, nur einmal gezählt, sodass auf Universitätsebene 596 Artikel in die Analyse miteinbezogen wurden. Für die Auswertung der einzelnen Organisationseinheiten (Ebene der Departments) wurde die Mehrfachzählungen beibehalten, d.h. ein Artikel der von Autor/inn/en zweier oder mehrerer Departments gemeinsam verfasst wurde, wird bei jedem der beteiligten Departments gezählt.

Bei der Auswertung wurden für jeden Artikel die Merkmale Verlag, Anzahl der WU-Autor/inn/en und WU-Zu-

15 Zum Datensatz siehe Seyffertitz und Katzmayr (2021). 


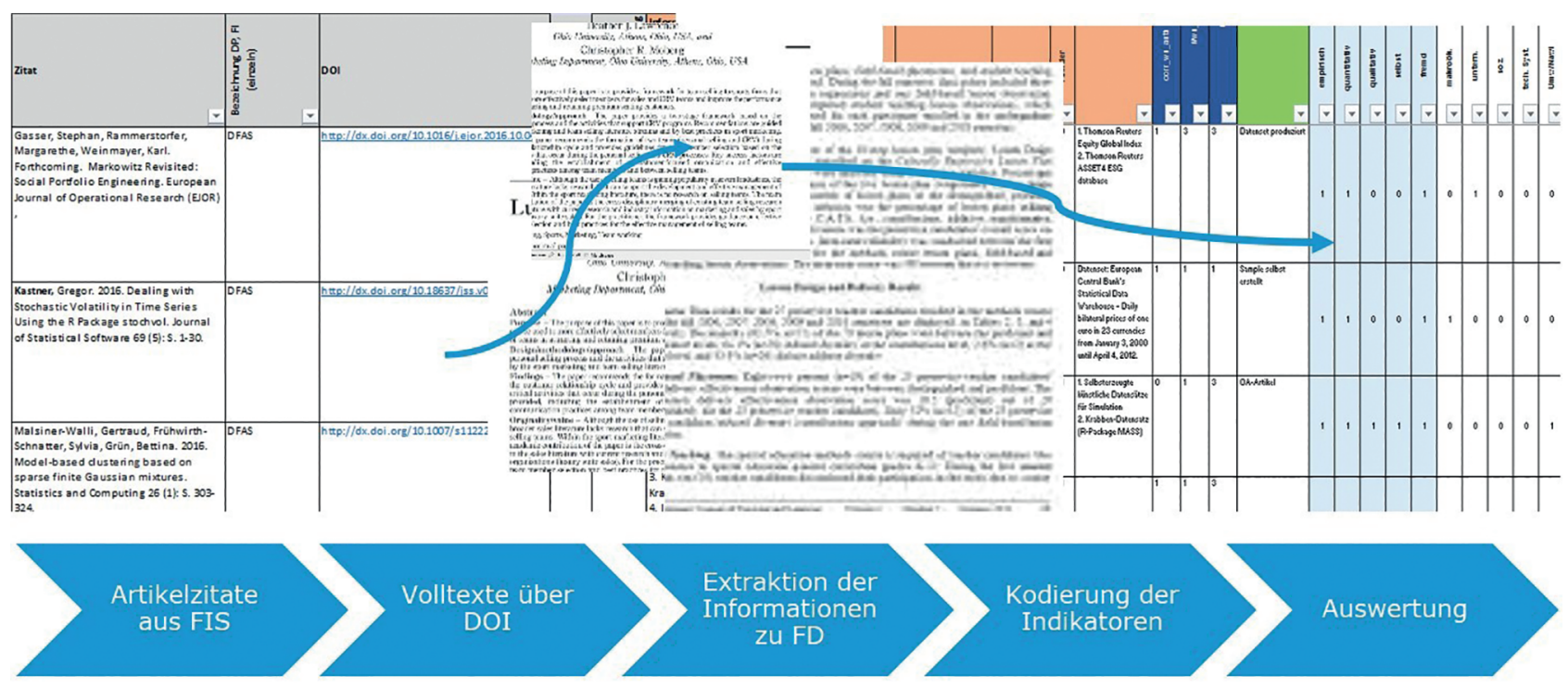

Abb. 1: Schematische Darstellung der Dokumentenanalyse

gehörigkeit des/r korrespondierenden Autor/s/in, Gesamtzahl der Autor/inn/en und das etwaige Vorliegen eines Fördergebers erhoben. Weiters wurden Informationen zur Herkunft der Daten, deren Eigenschaften (quantitative und/oder qualitative Daten), und deren Format bzw. der Datentyp erhoben (siehe Abb. 1).

\subsection{Qualitative Analyse: Semi-strukturierte Interviews}

Praktiken und Sichtweisen von Forschenden in Bezug auf ihre Forschungsdaten existieren hauptsächlich in Form von intrinsischem oder implizitem Wissen. Diese Art von Wissen ist von Natur aus wenig strukturiert. Qualitative Methoden bieten eine effektive Möglichkeit, dieser Art von Wissen nachzuspüren und in Folge $\mathrm{zu}$ analysieren. Es wurden semi-strukturierte Interviews durchgeführt, da diese Erhebungsform besonders geeignet ist, um qualitative, beschreibende und detaillierte Daten zu erheben, die eng mit dem Lebens- und Arbeitsumfeld der Befragten verbunden sind. Die Durchführung dieser Art von Interviews ist eine der am weitesten verbreiteten Techniken, um ein ganzheitliches Verständnis der Standpunkte und Meinungen der Teilnehmer/innen zu einem bestimmten Thema zu erlangen. ${ }^{16}$

Die Befragung verfolgte also das Ziel, das zuvor bei einer quantitativen Dokumentenanalyse gewonnene Bild der Forschungsdatenlandschaft der WU durch einen Ein-

16 Vgl. Pickard und Childs (2013), Yin (2018). blick in den tatsächlichen Umgang der Forschenden mit ihren Daten entlang des sogenannten Lebenszyklus von Forschungsdaten anzureichern. Dieser Lebenszyklus umspannt alle Phasen der Generierung und Verwendung von Forschungsdaten über die Planung des Forschungsprojektes über die Datenerhebung bzw. -generierung, Analyse und Verarbeitung der Daten, Publikation der Forschungsergebnisse bis hin zur eventuellen Nachnutzung und Langzeitarchivierung. ${ }^{17}$ Zusätzlich waren im verwendeten Gesprächsleitfaden auch Fragen zu möglichen bzw. erwünschten Services rund um FDM enthalten. ${ }^{18}$ Ein weiterer Vorteil dieser Erhebungsmethode ist strategischer Natur und bezieht sich auf die Positionierung der Bibliothek innerhalb der Universität: Durch den persönlichen Kontakt mit den Forschenden konnten nicht nur die benötigten Informationen erhoben werden, sondern es konnte darüber hinaus auch vermittelt werden, dass in der Bibliothek Kompetenz und Bereitschaft besteht, sich dieses Themas anzunehmen.

Vor der Zusammenstellung der Samples der Befragten interessiert zunächst die Art des Wissens, das zur Beantwortung der Forschungsfrage erforderlich ist. Eine Expertin bzw. ein Experte verfügt über „technisches, Prozess- und Deutungswissen, das sich auf sein spezifisches professionelles oder berufliches Handlungsfeld bezieht. Insofern besteht das Expertenwissen nicht allein aus systematisiertem, reflexiv zugänglichem Fach- oder Sonderwissen, sondern es weist zu großen Teilen den Charakter

17 Vgl. van den Eynden (2020).

18 Siehe Katzmayr und Seyffertitz (2019). 
von Praxis- oder Handlungswissen auf““. ${ }^{19}$ Das primäre Auswahlkriterium war daher das Vorliegen praktischer Erfahrung mit Forschungsdaten, die in der Veröffentlichung datenbasierter Forschungsergebnisse resultiert. Die quantitative Dokumentenanalyse lieferte demnach den Ausgangspunkt für die Rekrutierung der Befragten. Schließlich wurden 25 Interviews mit Forschenden aus allen akademischen Fachbereichen der WU durchgeführt. Die Interviews dauerten durchschnittlich eine Stunde und wurden bis auf eine Ausnahme aufgezeichnet.

Die Analyse der Interviews kann als ein Prozess der zunehmenden Verdichtung des Materials beschrieben werden. ${ }^{20}$ Konkret wurde in vier Schritten ausgewertet: Erstens wurden die aufgezeichneten Interviews paraphrasiert, d.h., der Inhalt des Gesprächs wurde der Chronologie des Gesprächsverlaufs folgend wiedergegeben. In einem zweiten Schritt wurden die thematisch zusammengehörenden Passagen eines Interviews zusammengestellt. Der dritte Schritt der Verdichtung des Materials bestand darin, bei der Auswertung die Ebene des einzelnen Interviews zu verlassen und thematisch zusammengehörende Passagen in einem neuen Dokument zusammenzustellen. Schließlich wurde viertens das gemeinsame Wissen der Befragten von den konkreten Gesprächsverläufen abgelöst und verallgemeinert. Die so gewonnenen Ergebnisse werden in Abschnitt 4.2 beschrieben.

\section{Ergebnisse}

\subsection{Dokumentenanalyse}

Von den 596 ausgewerteten Artikeln (Einzelzählung auf Universitätsebene, im weiteren Text als WU-Ebene bezeichnet) enthielten 250 Artikel Forschungsdaten (42\%). Eine separate Auswertung der juristischen Departments ergab, dass Forschungsdaten im Sinne der oben genannten Definition in lediglich drei der 164 ausgewerteten Artikel vorkommen. Dies ist in erster Linie auf die Forschungstradition und Publikationskultur der juristischen Departments zurückzuführen. Infolgedessen sowie aufgrund der Tatsache, dass aus den Daten keine allgemein gültigen Schlussfolgerungen gezogen werden könnten, wurde auf eine explizite Auswertung der rechtswissenschaftlichen Departments verzichtet.

19 Bogner und Menz (2005) 46.

20 Das im Folgenden beschriebene Vorgehen ist angelehnt an Meuser und Nagel (2005).
Tab. 2 gibt einen ersten Überblick über die Variablen „Vorliegen von Forschungsdaten“, „Fördergeber“ und „Autorenschaft“ in den ausgewerteten Zeitschriftenartikeln. Auf WU-Ebene weisen 58 \% der 596 ausgewerteten Artikel keine Forschungsdaten auf. Ohne die beiden juristischen Departments (im weiteren Text als DP-Ebene bezeichnet) reduziert sich die Zahl der Artikel ohne Forschungsdaten von 346 auf 185. Somit finden sich in mehr als der Hälfte der Zeitschriftenpublikationen (57,18 \% von $\mathrm{n}=432$ ) Forschungsdaten. Lediglich rund $21 \%$ der Arbeiten wurden durch Fördergeber unterstützt (Drittmittel).

Tab. 2: Indikatoren Vorliegen von Forschungsdaten, Förderung und Autorenschaft auf WU-Ebene (596 Artikel ausgewertet) und auf DPEbene (432 Artikel ausgewertet)

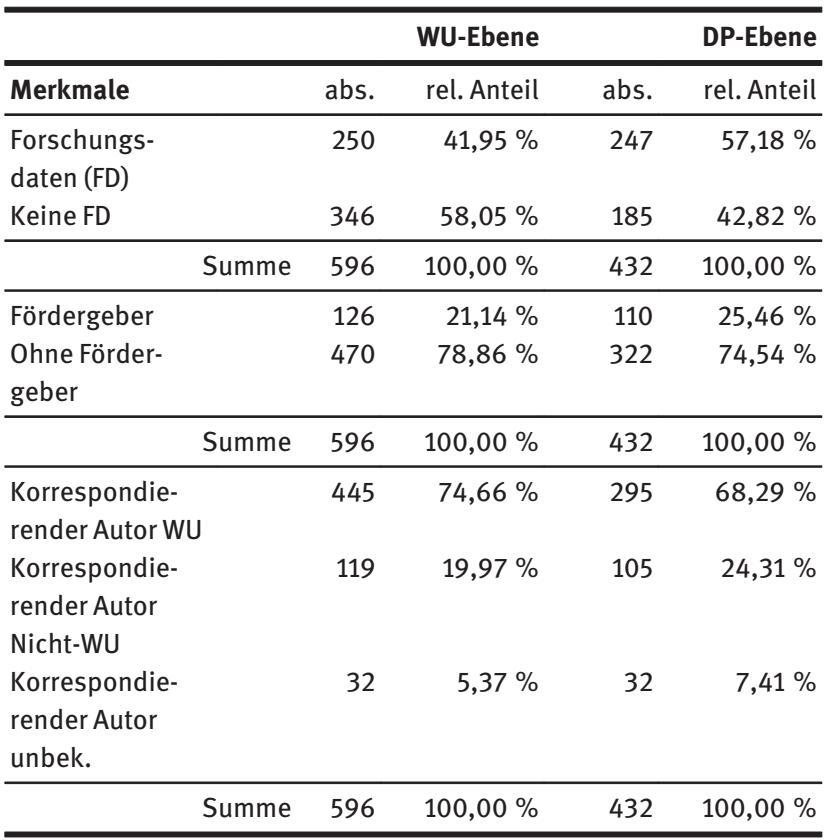

Fast $75 \%$ der insgesamt ausgewerteten Zeitschriftenartikel weisen WU-Forschende als korrespondierende Autor/inn/ en aus. In ca. $5 \%$ der Fälle (WU-Ebene) konnte die korrespondierende Autorenschaft nicht identifiziert werden. Interessant ist, dass der Anteil an Artikeln, die im Rahmen eines geförderten Forschungsprojektes entstanden sind, vergleichsweise gering ausfällt (ca. $21 \%, 126$ Artikel, s. Tab. 3). Weiters zeigt sich zum einen, dass zwei Drittel der geförderten Forschungsarbeiten Forschungsdaten aufweisen, und zum anderen, dass beinahe $65 \%$ aller Artikel, in denen keine Fördergeber genannt waren, keine Forschungsdaten aufweisen. Der Schluss, dass Fördergeber nur datenbasierte Forschungsprojekte fördern, lässt sich daraus nicht ableiten, da in den vorliegenden Zahlen (Tab. 3) auch die Forschungspublikationen der rechtswissenschaftlichen Departments inkludiert sind. 
Tab. 3: Forschungsförderung vs. Forschungsdaten auf WU-Ebene (596 ausgewertete Artikel)

\begin{tabular}{lrrr}
\hline & $\begin{array}{r}\text { Forschungs- } \\
\text { daten }\end{array}$ & $\begin{array}{r}\text { Keine } \\
\text { Forschungs- } \\
\text { daten }\end{array}$ & $\begin{array}{r}\text { Summe geför- } \\
\text { dert/nicht } \\
\text { gefördert }\end{array}$ \\
\hline Förderung & 84 & 42 & 126 \\
Keine Förderung & 166 & 304 & 470 \\
\hline Summe & 250 & 346 & 596 \\
\hline
\end{tabular}

Betrachtet man die Datenformen (qualitativ/quantitativ), so zeigt sich, dass insgesamt Daten quantitativer Natur in den empirischen Arbeiten dominieren (vgl. Tab. 4). In $86 \%$ der Arbeiten mit Forschungsdaten liegen quantitative Daten vor, in etwa $30 \%$ kommen beide Datenformen vor. Der entsprechende Anteil, wo lediglich qualitative Forschungsdaten vorliegen, liegt bei knapp $14 \%$.

Tab. 4: Auftreten von Forschungsdaten in den Zeitschriftenartikeln nach Datenform auf WU- und DP-Ebene

\begin{tabular}{lrrrr}
\hline Datenform & \multicolumn{2}{c}{ WU-Ebene $(\mathrm{n}=250)$} & \multicolumn{2}{c}{ DP-Ebene $(\mathrm{n}=247)$} \\
\hline & abs. & rel. Anteil & abs. & rel. Anteil \\
\hline nur quantitative FD & 141 & $56,40 \%$ & 139 & $56,28 \%$ \\
nur qualitative FD & 35 & $14,00 \%$ & 34 & $13,77 \%$ \\
beide Formen & 74 & $29,60 \%$ & 74 & $29,96 \%$ \\
\hline & 250 & $100,00 \%$ & 247 & $100,00 \%$ \\
\hline
\end{tabular}

Beim inhaltlichen Ursprung der Forschungsdaten (Datendomänen) liegt der Schwerpunkt bei Daten, die ihren Ursprung in sozialen Phänomenen und sozialen Interaktionen sowie Wirtschafts- und Unternehmensdaten haben (Tab. 5). Daten technischer Systeme spielen hier eine untergeordnete Rolle. Bei fast $23 \%$ der Artikel stammen die Forschungsdaten aus mehr als einer Domäne, wobei mit fast $19 \%$ die Artikel, in welchen Daten aus zwei verschiedenen Domänen vorliegen, dominieren.
Tab. 5: Absolute und relative Häufigkeiten der einzelnen Datendomänen in den empirischen Artikeln auf WU-Ebene $(n=250)$ und auf DP-Ebene $(n=247)$

\begin{tabular}{|c|c|c|c|c|}
\hline \multirow[t]{2}{*}{ Datendomänen } & \multirow{2}{*}{$\begin{array}{r}\text { WU-Ebene } \\
\text { abs. }\end{array}$} & \multicolumn{3}{|c|}{ DP-Ebene } \\
\hline & & rel. & abs. & rel. \\
\hline Wirtschaftsdaten & 65 & $20,38 \%$ & 63 & $19,94 \%$ \\
\hline Unternehmensdaten & 56 & $17,55 \%$ & 56 & $17,72 \%$ \\
\hline $\begin{array}{l}\text { Soziale Phänomene } \\
\& \text { Interaktionen }\end{array}$ & 97 & $30,41 \%$ & 97 & $30,70 \%$ \\
\hline $\begin{array}{l}\text { Daten technischer } \\
\text { Systeme }\end{array}$ & 11 & $3,45 \%$ & 11 & $3,48 \%$ \\
\hline $\begin{array}{l}\text { Daten im umwelt- } \\
\text { bzw. naturwissen- } \\
\text { schaftlichen Kontext }\end{array}$ & 15 & $4,70 \%$ & 15 & $4,75 \%$ \\
\hline Sonstige & 75 & $23,51 \%$ & 74 & $23,42 \%$ \\
\hline $\begin{array}{l}\text { Summe Häufigkeit } \\
\text { des Auftretens über } \\
\text { alle Artikel mit FD }\end{array}$ & 319 & $100,00 \%$ & 316 & $100,00 \%$ \\
\hline
\end{tabular}

Anm.: Gleichzeitiges Auftreten verschiedener Ausprägungen in der jeweiligen Publikation möglich.

Betrachtet man das Merkmal Datenformattypen (Tab. 6), so treten Forschungsdaten am häufigsten in alphanumerischer Form auf, d.h. Zahlen und/oder Text. Da für den ursprünglich vorgesehenen Datentyp „Code/Software" keine konstant validen Daten aus den Zeitschriftenartikeln abgeleitet werden konnten (teilweise konnte nur aus dem Kontext auf das Vorliegen von Software oder Code geschlossen werden) wurden diese unter der Rubrik „alphanumerische Daten“ kategorisiert. Meist traten die Daten in Form von R-Code oder auch als Pseudocode zur Darstellung von Algorithmen auf.

Tab. 6: Absolute und relative Häufigkeiten der einzelnen Datenformattypen in den Artikeln mit Forschungsdaten auf WU-Ebene $(n=250)$ und auf DP-Ebene $(n=247)$

\begin{tabular}{lrrrr}
\hline Datenformattypen & WU-Ebene & \multicolumn{3}{c}{ DP-Ebene } \\
\hline & abs. & rel. & abs. & rel. \\
\hline Bild & 9 & $3,04 \%$ & 9 & $3,07 \%$ \\
Audio & 33 & $11,15 \%$ & 33 & $11,26 \%$ \\
Video & 4 & $1,35 \%$ & 4 & $1,37 \%$ \\
Alphanumerische & 249 & $84,12 \%$ & 246 & $83,96 \%$ \\
(inkl. Software) & 1 & $0,34 \%$ & 1 & $0,34 \%$ \\
Sonstige & 296 & $100,00 \%$ & 293 & $100,00 \%$ \\
\hline $\begin{array}{l}\text { Gesamthäufigkeit } \\
\text { über alle Artikel mit FD }\end{array}$ & & & & \\
\hline
\end{tabular}

Anm.: Gleichzeitiges Auftreten verschiedener Ausprägungen in der jeweiligen Publikation möglich 
Betrachtet man die einzelnen Departments (ohne rechtswissenschaftliche Departments), zeigt sich, dass Volkswirtschaft und Sozioökonomie mit jeweils über $17 \% \mathrm{mehr}$ als ein Drittel der Artikel mit Forschungsdaten stellen (Tab. 7). Innerhalb eines einzelnen Departments ist bei Informationsverarbeitung und Prozessmanagement der Anteil an Artikeln, in welchen Forschungsdaten auftreten, mit fast $85 \%$ am höchsten (Tab. 8).

Tab. 7: Verteilung der Zeitschriftenartikel mit Forschungsdaten $(n=265)$ auf die Departments

\begin{tabular}{|c|c|c|}
\hline Department & $\begin{array}{r}\text { Artikel mit FD } \\
\text { absolut }\end{array}$ & $\begin{array}{r}\text { Anteil an } \\
\text { Gesamtzahl der } \\
\text { Artikel mit FD }\end{array}$ \\
\hline $\begin{array}{l}\text { DP Finance, Accounting and } \\
\text { Statistics }\end{array}$ & 34 & $12,83 \%$ \\
\hline $\begin{array}{l}\text { DP Informationsverarbeitung } \\
\text { und Prozessmanagement }\end{array}$ & 27 & $10,19 \%$ \\
\hline DP Management & 32 & $12,08 \%$ \\
\hline DP Marketing & 22 & $8,30 \%$ \\
\hline DP Sozioökonomie & 46 & $17,36 \%$ \\
\hline DP Strategy and Innovation & 14 & $5,28 \%$ \\
\hline DP Volkswirtschaft & 47 & $17,74 \%$ \\
\hline DP Welthandel & 21 & $7,92 \%$ \\
\hline \multicolumn{3}{|l|}{ DP Fremdsprachliche } \\
\hline Wirtschaftskommunikation & 8 & $3,02 \%$ \\
\hline Sonstige Zugehörigkeit & 14 & $5,28 \%$ \\
\hline & Summe & $100,00 \%$ \\
\hline
\end{tabular}

Tab. 8: Anteil der Zeitschriftenartikel mit FD an allen ZS-Artikeln innerhalb des jeweiligen Departments

\begin{tabular}{|c|c|c|c|}
\hline Department & $\begin{array}{l}\text { Artikel mit } \\
\text { FD im DP }\end{array}$ & $\begin{array}{l}\text { Artikel im } \\
\text { DP gesamt }\end{array}$ & $\begin{array}{r}\text { Anteil der } \\
\text { Artikel mit } \\
\text { FD an allen } \\
\text { Artikeln im } \\
\text { DP }\end{array}$ \\
\hline $\begin{array}{l}\text { DP Finance, Accounting and } \\
\text { Statistics }\end{array}$ & 34 & 94 & $36,17 \%$ \\
\hline $\begin{array}{l}\text { DP Informationsverarbei- } \\
\text { tung und Prozess- } \\
\text { management }\end{array}$ & 27 & 32 & $84,38 \%$ \\
\hline DP Management & 32 & 44 & $72,73 \%$ \\
\hline DP Marketing & 22 & 57 & $38,60 \%$ \\
\hline DP Sozioökonomie & 46 & 75 & $61,33 \%$ \\
\hline DP Strategy and Innovation & 14 & 21 & $66,67 \%$ \\
\hline DP Volkswirtschaft & 47 & 62 & $75,81 \%$ \\
\hline DP Welthandel & 21 & 29 & $72,41 \%$ \\
\hline DP Fremdsprachliche & 8 & 16 & $50,00 \%$ \\
\hline Wirtschaftskommunikation & & 21 & \\
\hline Sonstige Zugehörigkeit & 14 & & $66,67 \%$ \\
\hline & 265 & 451 & \\
\hline
\end{tabular}

Ein Blick auf den Indikator Datendomänen (Tab. 9) zeigt im Department Management einen verhältnismäßig hohen Anteil an Arbeiten mit „Daten sozialer Phänomene bzw. Interaktionen“, während die ausgewerteten Artikel des Departments Volkswirtschaft von einem hohen Anteil an „Wirtschaftsdaten“ geprägt sind. Die ausgewerteten Artikel zeigen auch, dass in den verschiedenen Arbeiten immer wieder Daten aus verschiedenen Domänen erhoben, verarbeitet oder kombiniert werden.

Tab. 9: Auftreten von Forschungsdaten in den Zeitschriftenartikeln $(n=265)$ nach Datendomänen auf DP-Ebene (Absolutwerte)

\begin{tabular}{|c|c|c|c|c|c|c|}
\hline Department & $\begin{array}{l}\text { Wirt- } \\
\text { schaft- } \\
\text { sdaten }\end{array}$ & $\begin{array}{l}\text { Unter- } \\
\text { neh- } \\
\text { mens- } \\
\text { daten }\end{array}$ & $\begin{array}{r}\text { Daten } \\
\text { soz. } \\
\text { Phäno- } \\
\text { mene } \\
\text { bzw. In- } \\
\text { terak- } \\
\text { tionen }\end{array}$ & $\begin{array}{l}\text { Daten } \\
\text { techn. } \\
\text { Syste- } \\
\text { me }\end{array}$ & $\begin{array}{r}\text { Daten } \\
\text { im na- } \\
\text { turw./ } \\
\text { um- } \\
\text { welt- } \\
\text { wiss. } \\
\text { Kontext }\end{array}$ & $\begin{array}{r}\text { Sonsti- } \\
\text { ge }\end{array}$ \\
\hline $\begin{array}{l}\text { DP Finance, } \\
\text { Accounting } \\
\text { and Statistics }\end{array}$ & 14 & 12 & 6 & 0 & 3 & \\
\hline $\begin{array}{l}\text { DP Informati- } \\
\text { onsverarbei- }\end{array}$ & 0 & 0 & 12 & 7 & 1 & \\
\hline
\end{tabular}

tung und

Prozessmana-

gement

DP Manage-

ment

$\begin{array}{lllllll}\text { DP Marketing } & 0 & 6 & 15 & 0 & 1 & 5\end{array}$

$\begin{array}{lllllll}\text { DP Sozioöko- } & 23 & 5 & 19 & 3 & 11 & 18\end{array}$

nomie

$\begin{array}{lllllll}\text { DP Strategy } & 0 & 2 & 10 & 0 & 0 & 6\end{array}$

and Innova-

tion

$\begin{array}{lllllll}\text { DP Volkswirt- } & 30 & 8 & 10 & 0 & 2 & 9\end{array}$

schaft

$\begin{array}{lllllll}\text { DPWelt- } & 2 & 18 & 3 & 0 & 0 & 2\end{array}$

handel

DP Fremd-

sprachliche

Wirtschafts-

kommunika-

tion

Sonstige

Zugehörigkeit

$\begin{array}{lllllll}\text { Summe } & 74 & 59 & 104 & 11 & 15 & 78\end{array}$

Auftrittshäu-

figkeit

Anm.: Gleichzeitiges Auftreten verschiedener Ausprägungen in der jeweiligen Publikation möglich.

Betreffend Datenformattypen ist der alphanumerische Typ der am häufigsten Auftretende. Praktisch in jeder Arbeit sind Daten in Form von Zahlen, Texten (strukturiert oder 
unstrukturiert) oder Software-Codes (z. B. R) anzutreffen (Tab. 10). Audiobasierte Forschungsdaten finden sich überwiegend in den Departments Management, Sozioökonomie sowie Informationsverarbeitung und Prozessmanagement. Insgesamt stellen Publikationen, worin bild- oder videobasierte Forschungsdaten vorkommen, einen eher geringen Anteil dar.

Tab. 10: Auftreten von Forschungsdaten in den Zeitschriftenartikeln $(n=265)$ nach Datenformattypen auf DP-Ebene (Absolutwerte)

\begin{tabular}{lcccrr}
\hline Department & Bild & Audio & Video & $\begin{array}{r}\text { Alpha- } \\
\text { num. }\end{array}$ & $\begin{array}{r}\text { Sonsti- } \\
\text { ge }\end{array}$ \\
\hline $\begin{array}{l}\text { DP Finance, Accounting } \\
\text { and Statistics }\end{array}$ & 0 & 0 & 0 & 34 & 0 \\
$\begin{array}{l}\text { DP Informationsver- } \\
\text { arbeitung und }\end{array}$ & 1 & 6 & 1 & 27 & 0 \\
Prozessmanagement & & & & & \\
DP Management & 2 & 9 & 1 & 31 & 1 \\
$\begin{array}{l}\text { DP Marketing } \\
\text { DP Sozioökonomie }\end{array}$ & 2 & 2 & 0 & 22 & 0 \\
$\begin{array}{l}\text { DP Strategy and } \\
\text { Innovation }\end{array}$ & 0 & 4 & 0 & 46 & 0 \\
$\begin{array}{l}\text { DP Volkswirtschaft } \\
\text { DP Welthandel }\end{array}$ & 1 & 0 & 0 & 14 & 0 \\
$\begin{array}{l}\text { DP Fremdsprachliche } \\
\text { Wirtschaftskommuni- }\end{array}$ & 0 & 1 & 0 & 21 & 0 \\
kation & 3 & 2 & 8 & 0 \\
Sonstige Zugehörigkeit & 1 & 2 & 0 & 14 & 0 \\
\hline Summe Auftrittshäufig- & 9 & 35 & 4 & 260 & 1 \\
keit & & & & & \\
\hline
\end{tabular}

Anm.: Gleichzeitiges Auftreten verschiedener Ausprägungen in der jeweiligen Publikation möglich.

Zusammenfassend zeigt sich, dass zwei Drittel der Arbeiten mit Forschungsdaten auf die fünf Departments Volkswirtschaft, Sozioökonomie, Finance, Accounting \& Statistics, Management und Informationsverarbeitung und Prozessmanagement entfallen. Grundsätzlich dominieren dabei quantitative Daten, vor allem bei Management und in der Sozioökonomie. Bei den Datendomänen dominieren Daten der Kategorie „Wirtschaftsdaten“ und „Unternehmensdaten“, die zusammen gut $50 \%$ ausmachen. Artikel mit Daten „Sozialer Phänomene und Interaktion“ sind eher qualitativer Natur und liegen in ca. 38 \% der Fälle vor. Der dominierende Datentyp ist der Typ „alphanumerische Daten“. Daten dieses Typs liegen meist in Form von Zahlen und Texten, in strukturierter und unstrukturierter Form bzw. Tabellenblättern vor.

\subsection{Semi-strukturierte Interviews}

In diesem Abschnitt werden die wichtigsten Ergebnisse der 25 Interviews präsentiert. Da die Erlangung eines umfassenden Bildes des Forschungsdatenmanagements der WU im Vordergrund steht, werden isolierte oder einzelne Aussagen, die nicht zu dieser umfassenderen Sichtweise beigetragen haben, im Folgenden nicht dargestellt.

\subsubsection{Datenmanagementpläne (DMP) und Vorgaben von Fördergebern}

Nur rund die Hälfte der Befragten hatte schon mindestens einmal die Erfahrung gemacht, dass Fördergeber besondere Anforderungen bezüglich Forschungsdaten stellten. Eine Minderheit der Forschenden befand sich in der Situation, einen DMP erstellen zu müssen. In diesen Fällen wurden die Deutsche Forschungsgemeinschaft (DFG), das European Research Council (ERC), das Economic and Social Research Council (ESRC) und das Programm Horizon 2020 der Europäischen Kommission genannt.

\subsubsection{Trends und Entwicklungen hinsichtlich Forschungsdaten}

Hier ergibt sich naturgemäß eine große Abhängigkeit vom jeweiligen Forschungsgebiet. Als gemeinsame Herausforderung kann in den mit quantitativen Methoden forschenden Bereichen die steigende Bedeutung von großen Datenmengen bzw. Big Data gesehen werden. Damit verbunden ist der wachsende Fokus auf das World Wide Web und der Sozialen Medien als Datenquellen. Dies führt zu wachsenden Anforderungen sowohl an den Speicherplatz als auch an die Rechenkapazität.

\subsubsection{Beschreiben, Verwalten und Speichern von Forschungsdaten im Forschungsprozess}

Erwartungsgemäß zeigt sich hier ein sehr heterogenes Bild. Während des Forschungsprozesses liegen die Daten in den meisten Fällen verstreut (teilweise auf dem internen Server, auf externen Festplatten, privaten Notebooks etc.). Auch eine bewusst verfolgte Datenmanagementstrategie also ein konsistentes und planmäßiges Benennen, Ablegen und Verwalten von Daten über Forschungsgruppen und -projekte hinweg - stellt auf Instituts- oder Departmentebene eher die Ausnahme dar. Für den Datenaustausch werden häufig externe Cloud-Systeme verwendet - 
genannt wurden Dropbox, Google Drive und Amazon Web Services.

\subsubsection{Forschungsdaten im Publikationsprozess}

Quantitative Forschungsdaten betreffend wurde der überwiegende Teil der Befragten aus unterschiedlichen Disziplinen bereits mit Forschungsdaten-Policies von Zeitschriften oder Anforderungen von Gutachter/inne/n konfrontiert. Mehr als die Hälfte der Befragten hatte bereits konkrete Erfahrungen mit dem Veröffentlichen quantitativer Forschungsdaten gemacht. In zwei Fällen waren die Forschungsdaten und ihre Beschreibung das eigentliche Thema der Veröffentlichung (sog. „Data-Paper“).

Forschungsdaten, die im Rahmen der qualitativen empirischen Sozialforschung entstehen, wurden von den Befragten nicht publiziert, da diese Daten sehr kontextbezogen sind und die Reproduzierbarkeit von Forschungsergebnissen in diesem Feld nicht im Vordergrund steht. Zeitschriften machten hierbei auch keine Anforderungen.

\subsubsection{Langfristige Archivierung von Forschungsdaten}

Nach erfolgter Publikation findet in der Regel ein pragmatischer und fallbezogener Umgang mit Forschungsdaten statt. Weniger als ein Drittel der Befragten betreibt ein bewusstes, langfristiges Archivieren von Forschungsdaten, wobei unterschiedliche Herangehensweisen beschritten werden: externe Festplatten, spezielle Ordner auf dem internen Server oder das Betreiben eigener Webdatenbanken. Als Gründe, warum keine langfristige Archivierung erfolgt, werden insbesondere fehlende Notwendigkeit genannt (in manchen Disziplinen wie der Psychologie oder der Konsumentenforschung gelten Forschungsdaten nach der Publikation als „verbraucht“), sowie der hohe Aufwand, der für die hinreichende Beschreibung der Forschungsdaten betrieben werden muss.

\subsubsection{Erfahrungen mit Datenverlust}

Knapp die Hälfte der Befragten hatte bereits Erfahrungen mit Datenverlust. Üblicherweise betrafen die Verluste bereits verarbeitete Forschungsdaten, die nach z.T. erheblichem Zeitaufwand wiederhergestellt werden konnten. Allerdings: Trotz der eher uneinheitlichen und wenig planmäßigen Praktiken wurde ein unwiederbringlicher Datenverlust kaum berichtet.

\subsubsection{Teilen und Nachnutzen von Forschungsdaten}

Interessant in diesem Zusammenhang ist die Frage, inwieweit die Nachnutzung von Forschungsdaten in den an der WU vertretenen Disziplinen überhaupt von Belang ist. Die überwiegende Zahl der Befragten gab an, dass die Wiederverwendung bereits existierender Forschungsdaten durchaus relevant sein kann. In manchen Disziplinen ist dies wohl der Anforderung an Reproduzierbarkeit (quantitativer) Forschungsergebnisse geschuldet, aber auch sonst findet eine Wiederverwendung zumindest fallweise statt. Lediglich in der qualitativen empirischen Sozialforschung und in der Konsumentenforschung ist eine Nachnutzung zumindest von Primärdaten unüblich. Im letztgenannten Feld stellen allerdings Metastudien eine bedeutende Ausnahme von der herkömmlichen einmaligen Verwendung von Forschungsdaten dar, wodurch auch Zitationen für die ursprüngliche Publikation generiert werden.

Der Großteil der Befragten steht der Weitergabe von Daten nach erfolgter Erstpublikation grundsätzlich positiv gegenüber. Zumeist werden die Daten - falls rechtlich überhaupt möglich und inhaltlich als sinnvoll erachtet - ohne Auflagen weitergegeben. Fallweise ist die Weitergabe allerdings an Bedingungen geknüpft, v.a. Mitwirkung als Ko-Autor/in, wissenschaftliche Zusammenarbeit oder Informationen darüber, was mit den Forschungsdaten in weiterer Folge passiert bzw. was publiziert wird.

Unabhängig von der Bereitschaft der Befragten zur Nachnutzung wurden $u$.a. folgende Hemmnisse genannt, die dem Teilen von Forschungsdaten entgegenstehen:

- In einigen Forschungsdisziplinen können aufgrund des sehr speziellen Datenbedarfes Nachnutzungen grundsätzlich nicht stattfinden bzw. haben auch Replikationsstudien keine Tradition (letzteres insbesondere bei Verwendung qualitativer Forschungsdaten).

- Forschungsdaten stellen auch einen Wettbewerbsvorteil dar: Erst wenn die eigene Forschung anhand bestimmter Daten erschöpfend durchgeführt wurde, d.h. die Daten bei der Möglichkeit einer Mehrfachverwendung wissenschaftlich schon weitegehend verwertet wurden, wird Teilen zu einer Option.

- $\quad$ Es ist ein hoher Aufwand damit verbunden, Daten zum Teilen aufzubereiten, sei es, weil sie anonymisiert werden müssen oder weil umfangreiche Erläuterungen zum Verständnis der Daten notwendig sind.

Als Anreize, Daten zu teilen, können insbesondere gelten:

- Möglichkeit für wissenschaftliche Kooperationen und Mitwirkung als Ko-Autor/in, 
- Vermehrte Zitationen durch Metaanalysen und Replikationen.

\subsubsection{Policy für den Umgang mit Forschungsdaten an der WU}

Die Mehrzahl der Forschenden hatte eine neutrale bzw. abwägende Position zu einer Forschungsdaten-Policy, nur wenige standen ihr sehr positiv gegenüber oder wünschten sich diese. Drei inhaltlich zusammenhängende Beweggründe wurden häufiger genannt:

- In den an der WU vertretenen Disziplinen bestehen sehr unterschiedliche Herangehensweisen bezüglich Forschungsdaten. Eine Policy müsste darauf Rücksicht nehmen.

- $\quad$ Es gibt eine Sorge vor einer Überreglementierung bzw. zusätzlichen Bürokratisierung, d.h., dass zu viele praxisferne Regelungen den Forschungsalltag unnötig verkomplizieren.

- $\quad$ Es wird auf die zusätzliche Arbeitsbelastung für Forschende hingewiesen, um allfälligen neuen oder geänderten Richtlinien bzw. Anforderungen der WU in diesem Bereich zu entsprechen.

\subsubsection{Forschungsdatenmanagement als Service}

Die Antworten auf die Frage, welche gelungenen Ansätze im FDM an anderen Universitäten bekannt sind, waren wenig ergiebig. In einigen Fällen wurden die im angloamerikanischen Raum verbreiteten Ethikkommissionen (Ethical Clearance Committees) angesprochen: Diese regulieren u.a. auch den Prozess der Forschungsdatenerhebung und dessen Dokumentation und wurden eher als administrative Hürde wahrgenommen.

Im Hinblick auf eine mögliche Servicierung im Bereich FDM kristallisierten sich drei wesentliche Bedarfsfelder heraus:

- Informations- und Beratungsangebote für den Umgang mit Forschungsdaten: Der Bogen spannt sich hier von bewusstseinsbildenden Maßnahmen über die Einrichtung einer Anlaufstelle, der Beratung zu rechtlichen Fragestellungen und bei Projekteinreichungen (z.B. Datenmanagementplänen) bis hin zur Datenarchivierung. Trotz der eher negativen Wahrnehmung von Ethikkommissionen (siehe oben) wurde von einigen Forschenden der Bedarf nach einer ethischen Datenberatung bzw. Unterstützung beim ethischen Umgang mit Forschungsdaten genannt.
- (Langfristige) Archivierung von Forschungsdaten: Die Möglichkeit, Forschungsdaten sicher und ggf. auch dauerhaft zu speichern, stellt für viele Forschende, die keine institutseigenen Server oder Daten-Webseiten betreiben, ein wünschenswertes Service dar. Im Vordergrund dabei scheint eine einfache Handhabung dieser Services zu stehen.

- Technische Services: Hier wurden insbesondere Speicherkapazitäten und -möglichkeiten, Rahmenbedingungen für einen sicheren Datentransfer (auch zu internationalen Projektpartnern) bzw. Fernzugriff auf Daten genannt.

\section{Diskussion und Umsetzung}

\subsection{Zusammenfassung und Generalisierbarkeit der Ergebnisse}

Zusammenfassend zeigt sich, dass die Generierung und Verwendung von Forschungsdaten mit Ausnahme der Rechtswissenschaften in allen an der WU vertretenen Disziplinen von Bedeutung ist. Hinsichtlich Datensicherheit und Datenschutz scheinen sich die Forschenden in der Regel für den flexiblen Zugriff auf Daten und den Austausch von Informationen mit Kolleg/inn/en bei gleichzeitiger Minimierung des Verwaltungsaufwands zu interessieren. Es sollte daher darauf Bedacht genommen werden, dass Werkzeuge, Plattformen und Dienstleister, die gegenwärtig oder zukünftig zur Erhebung, Speicherung, Versionierung, Archivierung und zum Austausch von Daten herangezogen werden, im Einklang mit den neuen DSGVO-Richtlinien verwendet werden.

Die Verwendung von Forschungsdaten ist zwar nicht überall in gleichem Maße verbreitet, allerdings ist in allen Fächern ein Bedeutungszuwachs zu erwarten. Auffallend ist auch die große Vielfalt an Datendomänen und Datenformattypen: Obwohl wenig überraschend alphanumerische Daten aus dem Wirtschafts- und Unternehmensbereich sowie Daten zu sozialen Phänomenen und Interaktionen den Schwerpunkt an der WU ausmachen, sind auch Bild-, Audio- und Videodaten zu finden. Nach Departments haben die Volkswirtschaftslehre und die Sozioökonomie die höchste Datenaffinität.

Die Analyse der Forschungsergebnisse der WU zeigte eine vergleichsweise geringe Quote (rund $21 \%$ ) an Publikationen, die durch Drittmittel ermöglicht wurden. Dies $\mathrm{zu}$ wissen ist insofern wichtig, da eine niedrige Drittmittelquote in der Wahrnehmung der WU-Forschenden $\mathrm{zu}$ einem (scheinbar) geringeren Problemdruck und Handlungszwang im Umgang mit Forschungsdaten führen 
können. Darauf ist bei Beratungen von Forschenden Rücksicht zu nehmen.

Aus den empirischen Befunden lässt sich auch für die WU eine sogenannte Long-Tail-Verteilung der Forschungsdaten feststellen, d.h., dass eine ausgeprägte rechtsschiefe Verteilung der Datengröße über der Anzahl der Datensätze vorliegt. Anders gesagt: Neben einigen wenigen Big-DataProjekten, die an der WU insbesondere in den Datendomänen „Daten technischer Systeme“ sowie „Daten im Umwelt- bzw. naturwissenschaftlichen Kontext“" vorliegen, weist die weitaus überwiegende Anzahl an Datensätzen eine vergleichsweise geringe Größe auf. Diese Daten sind häufig das Produkt individueller, kleinerer oder wenig skalierter Forschungsprojekte und stellen gleichsam den wissenschaftlichen „Normalbetrieb“ in größeren Universitäten dar. ${ }^{21}$ Für die WU bedeutet das, dass die allermeisten Ansprüche an Services im FDM - zumindest hinsichtlich des Speicherplatzbedarfes - derzeit ohne große zusätzliche Infrastrukturen zu bewältigen sein sollte. Allerdings kann sich dies durch eine Änderung in den Forschungsschwerpunkten der WU künftig anders darstellen. Auch wurde die steigende Bedeutung von großen Datenmengen in den Interviews als genereller Trend genannt. Für eine einheitliche Beschreibung und Verwaltung der Forschungsdaten stellt diese Vielzahl an Datensätzen aus unterschiedlichsten Domänen jedoch eine Herausforderung dar.

Die Sonderstellung der Rechtswissenschaften betreffend die Verwendung bzw. Generierung von Forschungsdaten ist zum einen dem Methoden- bzw. Forschungsverständnis in dieser Disziplin geschuldet. Steht die sogenannte „Normtextarbeit“ im Vordergrund, also das hermeneutische Interpretieren von Rechtsnormen, so fallen definitionsgemäß keinen Forschungsdaten an, da publizierte wissenschaftliche Texte, Gesetzesmaterialien, Judikatur etc. gängigen Definitionen zufolge nicht als Forschungsdaten betrachtet werden. Anders verhält es sich bei der „Tatsachenarbeit“, also der Sachverhaltsermittlung und Beurteilung. ${ }^{22}$ Als Forschungsdaten in diesem Bereich können z. B. historische Urkunden, nichtveröffentlichte Urteile, Verträge und Gutachten, Stellungnahmen und Handreichungen für Geldgeber im Rahmen eines Projektes oder Ausschreibung sein etc. ${ }^{23}$ Da diese Form des empirischen Arbeitens an den rechtswissenschaftlichen Departments an der WU nicht im Vordergrund zu stehen scheint, ist die weitgehend fehlende Forschungsdatenverwendung in diesem Bereich erklärbar.

21 Vgl. Rice and Southall (2016) 28f.

22 Zum Verhältnis von Normtextarbeit versus Tatsachenarbeit siehe Kramer (2013) $37 \mathrm{f}$.

23 Vgl. Johannes (2017) 904.
Die Ergebnisse der empirischen Erhebung führten zur Umsetzung erster FDM-Maßnahmen an der WU (siehe unten Abschnitt 4.2). Hier zeigte sich, dass sich zwei der drei Handlungsfelder, die in den Interviews als vordringlich genannt wurden (Informations- und Beratungsangebote sowie langfristige Archivierung von Forschungsdaten) zumindest teilweise sehr gut in das Aufgabenportfolio der Universitätsbibliothek der WU einfügen lassen.

Da über den konkreten Fall hinaus Erkenntnisse aus einzelnen Fallstudien nur eine begrenzte theoretische Reichweite aufweisen, ist bei Generalisierungen Vorsicht angebracht. Hier verhält es sich ähnlich wie mit Experimenten in den Naturwissenschaften: Weitreichende Ergebnisse werden üblicherweise mittels mehrerer Experimente erzielt, die ein Phänomen unter unterschiedlichen Bedingungen zu replizieren versuchen. Die einzelne Fallstudie dient - wie das einzelne Experiment - eher dazu, bestehende Wissensbestände $\mathrm{zu}$ bestätigen, zu erweitern bzw. neue Themen oder Fragestellungen aufzuwerfen. ${ }^{24}$

Dies kann am Beispiel der bereits dargestellten Sonderstellung der Rechtswissenschaften betreffend die Forschungsdatenverwendung an der WU illustriert werden: so interessant und relevant dieser Sachverhalt für die Ausgestaltung lokaler FDM-Services auch ist, so ist er sicher nicht ohne weiteres auf andere Hochschulen übertragbar. Es wäre allerdings lohnend zu sehen, ob an anderen Hochschulen ähnliches zum Vorschein kommt und man vielleicht hinsichtlich Forschungsdatenverwendung generell von einer Eigenheit der Rechtswissenschaften im deutschsprachigen Raum sprechen kann.

Dies gilt in vergleichbarer Form auch für die anderen der hier dargestellten Ergebnisse. Jedenfalls übertragbar scheint uns die grundsätzliche methodische Vorgangsweise zu sein, mit der wir die Verwendung von Forschungsdaten an der WU erhoben haben. Diese Vorgangsweise haben wir in ein konzeptionelles Modell überführt, das weiter unten (Abschnitt 4.3) dargestellt wird.

\subsection{Umsetzung}

Im Zuge der Einrichtung des FDM an der WU wurden folgende Maßnahmen als prioritär empfohlen und unter Federführung der Universitätsbibliothek mittlerweile bereits umgesetzt:

- Verabschiedung einer Forschungsdaten-Policy: Um den Rahmen für weitere Aktivitäten in Bereich FDM aufzubereiten und um den einschlägigen Anforderun-

24 Vgl. Yin (2018) $20 \mathrm{f}$. 
gen externer Anspruchsgruppen (insbesondere Fördergeber und dem zuständigen Ministerium) besser begegnen zu können, wurde von der Universitätsbibliothek eine Policy für Forschungsdatenmanagement an der WU entworfen und von der Universitätsleitung verabschiedet. Das in den Interviews zur Sprache gekommene Gebot der Berücksichtigung unterschiedlicher Forschungskulturen wurde durch eine weitgehend offene und - was Regelungen betrifft zurückhaltende Rahmen-Policy, die von den einzelnen Departments konkretisiert werden kann, versucht zu gewährleisten. Im Vorfeld der Formulierung und Verabschiedung fanden Informationsveranstaltungen in der Forschungskommission und im akademischen Mittelbau statt, um die Bedeutung der Thematik für die WU aufzuzeigen und um über die Interviews hinaus wichtige Anregungen und Ansprüche bei der Formulierung der Policy berücksichtigen zu können.

- Erstellung einer Webseite zum Thema Forschungsdaten: Es wurde auf der Webseite der Universitätsbibliothek eine Rubrik mit Basisinformationen zum Umgang mit Forschungsdaten eingerichtet, wo der gesamte Lebenszyklus - von der Antragstellung (Datenmanagementplan) über die Beschaffung/Erhebung (rechtliche Fragestellungen, DSGVO) bis hin zu Publikation und Archivierung (Überblick über einschlägige Repositorien) - abgebildet ist. Neben konkreten Ansprechpersonen der betroffenen Dienstleistungseinrichtungen wird auch auf weiterführende Informationen zu Fördergebern, Best Practices etc. verwiesen.

- Schaffung einer Anlauf- und Servicestelle für Beratungen und Auskünfte zu Forschungsdaten: Es wurde eine zentrale Anlaufstelle in der Universitätsbibliothek geschaffen, wo alle mit Forschungsdaten zusammenhängenden Anfragen entweder direkt beantwortet werden oder eine Weiterleitung an externe oder interne Auskunftspersonen (Rechtsabteilung, Forschungsservice, IT-Services, Fördergeber) erfolgt.

Eine weitere Empfehlung war die Errichtung einer Steuerungsgruppe für Forschungsdatenmanagement an der WU, um die Umsetzung der verschiedenen Maßnahmen zu koordinieren und zu begleiten. Die Entwicklung eines institutionellen FDM ist eine bereichsübergreifende Aufgabe. Neben den Forschenden der WU sind im Rahmen der Serviceerbringung auch Dienstleistungsbereiche wie Forschungsservice, IT-Services, Universitätsbibliothek und Rechtsabteilung betroffen. Eine formale Einsetzung einer Steuerungsgruppe wurde von den zuständigen Leitungsgremien nicht weiterverfolgt. Vertreter/innen aus den oben genannten Dienstleistungsbereichen haben sich in einer - von der Universitätsbibliothek koordinierten informellen „AG Forschungsdaten“ zusammengefunden, um Weiterentwicklungen und Anpassungen des FDM an der WU zu begleiten.

\subsection{Ableitung eines Vorgehensmodells im FDM}

Aus der vorliegenden Fallstudie lässt sich ein konzeptionelles Vorgehensmodell ableiten (Tab. 11), das grundsätzlich auch in anderen Forschungsinstitutionen anwendbar ist, und zwar insbesondere dort, wo noch wenig oder keine Kenntnis über die Forschungsdatenlandschaft vorliegt bzw. keine oder nur wenig Erfahrungen mit Forschungsdatenmanagement vorhanden sind. Das vorgeschlagene Modell unterscheidet vier Phasen, wobei jede Phase durch drei Komponenten definiert wird. Jeder der vier Phasen liegen Zielsetzungen zugrunde. Die Zielsetzungen der Folgephase resultieren dabei aus den Ergebnissen der Vorgängerphase (mit Ausnahme der Planungsphase, die sozusagen den Ausgangspunkt markiert). Die Inhaltskomponente beschreibt Themen oder inhaltliche Prozesse, die zur Zielerreichung notwendig sind. Die Komponente Methoden/Tätigkeiten enthält die Art und Weise wie die Themen der Inhaltskomponente realisiert werden.

Die Planungsphase umfasst Problemidentifikation, Definition des Vorhabens, Formulierung der Zielsetzung, Identifikation relevanter Stakeholder und Grobplanung der weiteren Arbeitsschritte im Rahmen des Projekts. Den inhaltlichen Kern der Erhebungs- und Analysephase bilden zwei einander komplementierende Komponenten:

1. Analyse des Forschungsoutputs, um Einblicke in die „Forschungsdatenlandschaft“ der Universität zu gewinnen (d.h., die Suche einer Antwort auf die Frage „Welche Forschungsdaten haben wir überhaupt?“), und

2. Einblick in den tatsächlichen Umgang der Forschenden mit ihren Daten entlang des sogenannten Forschungszyklus.

Die aus den Erhebungen gewonnen empirischen Daten werden in der Interpretations- und Diskussionsphase genutzt. Diese Phase beinhaltet die Ableitung von Handlungsempfehlungen sowie Vorbereitung von Entscheidungsgrundlagen, um mögliche Handlungsoptionen vorzubereiten. Die Entscheidungsgrundlagen und Handlungsempfehlungen werden den relevanten Gremien bzw. Stakeholdern (z. B. Vizerektorat für Forschung, Forschungskommission etc.) präsentiert und in diesen in aller 
Tab. 11: Schematische Darstellung des konzeptionellen Vorgehensmodells

\begin{tabular}{|c|c|c|c|}
\hline Phasen & Zielsetzungen & Inhalte & Methoden/Tätigkeiten \\
\hline \multirow[t]{3}{*}{ Planungsphase } & Festlegung der Projektziele & Problemstellung & $\begin{array}{l}\text { Gründung der } \\
\text { Projektarbeitsgruppe }\end{array}$ \\
\hline & Planung der nachfolgenden Phasen & mögliche Projektszenarien & Projektplanung \\
\hline & & Relevante Stakeholder & \\
\hline \multicolumn{4}{|l|}{$\downarrow$} \\
\hline \multirow[t]{2}{*}{$\begin{array}{l}\text { Erhebungs- und } \\
\text { Analysephase }\end{array}$} & $\begin{array}{l}\text { Kenntnis der Forschungsdatenlandschaft } \\
\text { an der Institution }\end{array}$ & $\begin{array}{l}\text { Forschungsoutput und } \\
\text { Forschungsschwerpunkte }\end{array}$ & $\begin{array}{l}\text { Dokumentenanalyse } \\
\text { (quantitative Komponente) }\end{array}$ \\
\hline & $\begin{array}{l}\text { Kenntnis des Umgangs und Erfahrungen mit } \\
\text { Forschungsdaten entlang des Forschungs- } \\
\text { zyklus, Erhebung von Bedarfen }\end{array}$ & $\begin{array}{l}\text { Datenmanagement bzw. } \\
\text { Datenkultur in den verschiedenen } \\
\text { wissenschaftlichen Fachbereichen }\end{array}$ & $\begin{array}{l}\text { Semi-strukturierte Interviews } \\
\text { (qualitative Komponente) }\end{array}$ \\
\hline \multicolumn{4}{|l|}{$\downarrow$} \\
\hline \multirow[t]{2}{*}{$\begin{array}{l}\text { Interpretations- u. } \\
\text { Diskussionsphase }\end{array}$} & Ableitung von Handlungsempfehlungen & $\begin{array}{l}\text { FDM-Maßnahmen und } \\
\text { FDM-Service-Portfolio }\end{array}$ & $\begin{array}{l}\text { Präsentation in den } \\
\text { Entscheidungsgremien }\end{array}$ \\
\hline & $\begin{array}{l}\text { Priorisierung und Auswahl von FDM-spezi- } \\
\text { fischen Maßnahmen und möglichen } \\
\text { Dienstleistungen }\end{array}$ & & Gremiale Entscheidungsfindung \\
\hline \multicolumn{4}{|l|}{$\downarrow$} \\
\hline \multirow[t]{3}{*}{ Umsetzungsphase } & Operationalisierung der Maßnahmen & FDM-Policy & $\begin{array}{l}\text { Modelle zur Entwicklung von } \\
\text { FDM-Policies }\end{array}$ \\
\hline & Planung + Festlegung eines Zeithorizontes & FDM-Kontaktpunkt & $\begin{array}{l}\text { Festlegung der Zuständigkeiten } \\
\text { und Änderungen in den Aufgaben- } \\
\text { profilen der Mitarbeitenden }\end{array}$ \\
\hline & & Information zu FDM & $\begin{array}{l}\text { Entwicklung von Web-Content, } \\
\text { Broschüren etc. }\end{array}$ \\
\hline
\end{tabular}

Regel entschieden. Jene Handlungsempfehlungen, deren Umsetzung beschlossen wurde, wird im Rahmen einer Umsetzungsphase priorisiert, geplant und operationalisiert. Die Dauer der Umsetzung variiert bei den einzelnen Maßnahmen: Beispielsweise lässt sich die Entwicklung einer Policy in der Regel rascher bewerkstelligen, als ein längerfristiges Schulungskonzept.

Obwohl die vorliegende Struktur dieses Modells einen geringen Komplexitätsgrad aufweist, kann der operative Aufwand - vor allem in der Erhebungs- und Analysephase - stark variieren. Dies hängt von mehreren Faktoren ab: Art und Anzahl der vertretenen Disziplinen in der Universität, Ausmaß des Forschungsoutputs bzw. Vorhandensein von Daten über den Forschungsoutput, Gestaltung der Kommunikations- und Ablaufprozesse, Anzahl der beteiligten Mitarbeitenden und nicht zuletzt von der in der Planungsphase $\mathrm{zu}$ formulierenden Zielsetzung. In der hier dargestellten Fallstudie dauerte der Gesamtprozess, begin- nend mit der Planungsphase Ende 2016, rund drei Jahre und war Ende 2019 weitgehend abgeschlossen.

\section{Schlussfolgerungen und Ausblick}

Eine wichtige Erkenntnis, die aus der vorliegenden Fallstudie gewonnen werden konnte, ist zum einen die Tatsache, dass die Kenntnis um die verwendeten bzw. vorhandenen Forschungsdaten eine sehr wesentliche Voraussetzung für einen zielgerichteten Aufbau von Forschungsdateninfrastruktur und -services ist. Zum anderen müssen die Forschungstraditionen und die fachspezifischen Publikationskulturen in den einzelnen Forschungsbereichen bekannt sein. Auch institutionelle Rahmenbedingungen und organisatorische Strukturen sind wesentliche Faktoren, die beim Aufbau und der Entwicklung eines institutionellen FDM zu berücksichtigen sind. Gerade die bei- 
den letztgenannten Faktoren schränken die unmittelbare Anwendbarkeit der hier entwickelten FDM-Services auf andere Universitäten und Hochschulen ein. Aufgrund sich dynamisch verändernder Forschungsschwerpunkte, Weiterentwicklung von Forschungsmethoden ebenso wie technische Entwicklungen und Digitalisierung erscheint es wichtig, die entwickelten Maßnahmen im Zeitablauf einer Überprüfung auf Anpassungserfordernisse zu unterziehen. Aus Sicht der Universitätsbibliothek der WU lässt sich das Resümee ziehen, dass durch die Erhebung nicht nur der Grundstein für ein institutionelles FDM gelegt werden konnte, sondern auch ein neues und zukunftsträchtiges Aufgabenfeld im Portfolio der Bibliothek verankert werden konnte.

Für Institutionen, die institutionelles FDM im Rahmen eines umfangreicheren institutionellen Strategieprozesses entwickeln wollen, bietet sich als Alternative zu dem hier präsentierten Vorgehensmodell das Research Infrastructure Self-Evaluation (RISE) Rahmenwerk..$^{25}$ Dies bedeutet, dass bei der Wahl eines Strategieentwicklungsmodells für FDM immer auch nationale Besonderheiten mitberücksichtigt werden sollten. Abgesehen von der Modellwahl und rechtlichen Vorgaben der Wissenschaftspolitik ist für einen erfolgreichen Einsatz und entsprechende Umsetzung die institutionelle Selbstverpflichtung („Commitment“) einer Universität ein wesentlicher Erfolgsfaktor, um den Herausforderungen eines adäquaten institutionellen Forschungsdatenmanagements gerecht zu werden.

\section{Literaturverzeichnis}

Arbeitsgruppe Forschungsdaten (2018): Forschungsdatenmanagement. Eine Handreichung. Potsdam: Deutsches GeoForschungsZentrum GFZ. Verfügbar unter https://doi.org/10.2312/allian zoa.029.

Bauer, Bruno; Ferus, Andreas; Gorraiz, Juan; Gründhammer, Veronika; Gumpenberger, Christian; Mühlegger, Johannes Michael et al. (2015): Forschende und ihre Daten. Ergebnisse einer österreichweiten Befragung - Report 2015. Version 1.2. Hg. v. Koordinationsbüro Projekt e-Infrastructures Austria. Wien. Verfügbar unter http://phaidra.univie.ac.at/o:407513.

Berman, Elizabeth (2017): An Exploratory Sequential Mixed Methods Approach to Understanding Researchers' Data Management Practices at UVM: Integrated Findings to Develop Research Data Services. In: Journal of eScience Librarianship, 6 (1), e1104. Verfügbar unter https://doi.org.10.7191/jeslib.2017.1104.

Bertelmann, Roland; Pfeiffenberger, Hans (2015): Forschungsdaten und Bibliotheken. In: Praxishandbuch Bibliotheksmanagement, hg. v. Rolf Griebel, Hildegard Schäffler, Konstanze Söllner und

25 Rans und Whyte (2017), für die deutsche Wissenschaftslandschaft wurde das Modell von Hartmann et al. (2019) adaptiert.
Eva Anne Frantz, 639-51. Berlin, München, Boston: De Gruyter Saur (Reference).

Bogner, Alexander; Menz, Wolfgang (2005): Das theoriegenerierende Experteninterview. Erkenntnisinteresse, Wissensformen, Interaktion. In: Das Experteninterview: Theorie, Methode, Anwendung, hg. v. Alexander Bogner, Beate Littig und Wolfgang Menz, 33-70. 2. Aufl. Wiesbaden: VS Verlag für Sozialwissenschaften.

Cox, Andrew M.; Verbaan, Eddy (2018): Exploring research data management. London: Facet.

e-Infrastructures Austria (2016): Muster für Forschungsdatenmanagement-Policy an österreichischen Forschungseinrichtungen. Verfügbar unter https://phaidra.univie.ac.at/o:459162.

Hartmann, Niklas K.; Jacob, Boris; Weiß, Nadin (2019): RISE-DE Referenzmodell für Strategieprozesse im institutionellen Forschungsdatenmanagement (Version 1.0). Verfügbar unter https://doi.org/10.5281/zenodo.3585556.

Jensen, Uwe (2019): Forschungsdaten und Forschungsdatenmanagement in den Sozialwissenschaften. In: Forschungsdatenmanagement sozialwissenschaftlicher Umfragedaten, hg. v. Uwe Jensen, Sebastian Netscher und Katrin Weller, 13-35. Verlag Barbara Budrich. Verfügbar unter https://doi.org/10.2307/j.ctv bkk1p8.5.

Johannes, Paul C. (2017): Forschungsdatenmanagement in der Rechtswissenschaft - Eine Betrachtung von außen nach innen. In: Die Öffentliche Verwaltung, 70 (21), 899-906.

Katerbow, Matthias; Kümmel, Christoph; Crispin, Julia; Kerremans, Daphné (2020): Digitaler Wandel in den Wissenschaften Impulspapier. Deutsche Forschungsgemeinschaft DFG. Bonn. Verfügbar unter https://doi.org/10.5281/zenodo.4191345.

Katzmayr, Michael; Seyffertitz, Thomas (2019): Leitfaden zur Erhebung zum Forschungsdatenmanagement. Verfügbar unter https://doi.org/10.25651/1.2019.0021.

Kramer, Ernst A. (2013): Juristische Methodenlehre. 4. Aufl. München: C. H. Beck.

Meuser, Michael; Nagel, Ulrike (2005): ExpertInneninterviews - vielfach erprobt, wenig bedacht: Ein Beitrag zur qualitativen Methodendiskussion. In: Das Experteninterview: Theorie, Methode, Anwendung, hg. v. Alexander Bogner, Beate Littig und Wolfgang Menz, 71-93. 2. Aufl. Wiesbaden: VS Verlag für Sozialwissenschaften.

Neuroth, Heike (2013): Brauchen wissenschaftliche Bibliotheken „Data Librarians“? - Pro. In: B.I.T.online, 16 (5), 392-93. Verfügbar unter http://www.b-i-t-online.de/heft/2013-05/kontr overs.pdf.

Pickard, Alison Jane; Childs, Sue (2013): Research methods in information. 2. Aufl. London: Facet.

Rans, Jonathan; Whyte, Angus (2017): Using RISE, the Research Infrastructure Self-Evaluation Framework. v1.1. Edinburgh: Digital Curation Centre. Verfügbar unter http://www.dcc.ac.uk/sites/d efault/files/documents/publications/UsingRISE_v1_1.pdf.

Rice, Robin Charlotte; Southall, John (2016): The data librarian's handbook. London: Facet.

Seyffertitz, Thomas; Katzmayr Michael (2021): Dokumentenanalyse wissenschaftlicher Zeitschriftenartikel an der Wirtschaftsuniversität Wien 2017. [Datensatz] Wien: AUSSDA. Verfügbar unter DOI: https://doi.org/10.11587/7P6VWS.

Summers, Scott; Bishop, Libby; van den Eynden, Veerle; Corti, Louise (2020): Legal and ethical considerations in sharing data. In: Managing and sharing research data: A guide to good practice, 
hg. v. Louise Corti, Veerle van den Eynden, Libby Bishop und Matthew Woollard, 160-95. 2. Aufl. Los Angeles: SAGE.

van den Eynden, Veerle (2020): The research data lifecycle. In: Managing and sharing research data: A guide to good practice, hg. v. Louise Corti, Veerle van den Eynden, Libby Bishop und Matthew Woollard, 33-43. 2. Aufl. Los Angeles: SAGE.

van den Eynden, Veerle; Corti, Louise (2020): The importance of managing and sharing research data. In: Managing and sharing research data: A guide to good practice, hg. v. Louise Corti, Veerle van den Eynden, Libby Bishop und Matthew Woollard, 1-32. 2. Aufl. Los Angeles: SAGE.

WU Wirtschaftsuniversität Wien (2020): Wissensbilanz 2019. Wien: Wirtschaftsuniversität Wien. Verfügbar unter https://www.wu.a c.at/fileadmin/wu/h/structure/about/publications/Wissensbil anz/WU_Wissensbilanz_2019_Web.pdf.

Yin, Robert K. (2018): Case study research and applications. Design and methods. 6. Aufl. Los Angeles: Sage.

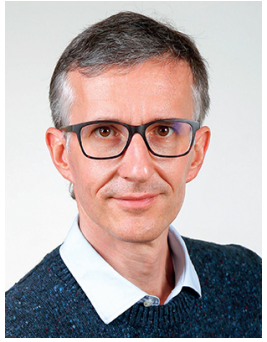

\section{Michael Katzmayr}

Wirtschaftsuniversität Wien

Universitätsbibliothek

Welthandelsplatz 1

A-1020 Wien

Österreich

michael.katzmayr@wu.ac.at

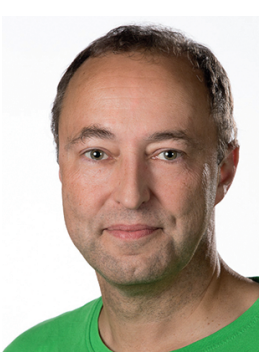

Thomas Seyffertitz

Wirtschaftsuniversität Wien

Universitätsbibliothek

Welthandelsplatz 1

A-1020 Wien

Österreich

thomas.seyffertitz@wu.ac.at 